\title{
Uniform Convergence of Discrete Curvatures from Nets of Curvature Lines
}

\author{
Ulrich Bauer • Konrad Polthier • Max Wardetzky
}

Received: 6 October 2008 / Revised: 12 December 2009 / Accepted: 13 December 2009 /

Published online: 12 January 2010

(C) The Author(s) 2010. This article is published with open access at Springerlink.com

\begin{abstract}
We study discrete curvatures computed from nets of curvature lines on a given smooth surface and prove their uniform convergence to smooth principal curvatures. We provide explicit error bounds, with constants depending only on properties of the smooth limit surface and the shape regularity of the discrete net.
\end{abstract}

Keywords Discrete curvatures · Polyhedral surfaces · Cotangent formula · Curvature lines

\section{Introduction}

The field of discrete differential geometry has brought to light intriguing discrete counterparts of classical differential geometric concepts and efficient geometric algorithms (see, e.g., [6, 14]). One aspect of this theory is convergence: classical smooth notions should arise in the limit of refinement. Recently, several convergence results have been obtained for curvatures and differential operators defined on polyhedral surfaces. Roughly, one may distinguish three approaches: (i) polynomial surface approximation (see, e.g., [7, 17]), (ii) geometric measure theory (see, e.g., [8, 13]), and (iii) finite element analysis (see, e.g., $[11,15]$ ). Among these, (i) provides pointwise convergent curvatures for many, but not all, discrete meshes. In contrast, (ii) and

\footnotetext{
U. Bauer $(\bowtie) \cdot$ M. Wardetzky

Institute for Numerical and Applied Mathematics, University of Göttingen, Lotzestr. 16-18, 37083 Göttingen, Germany

e-mail: bauer@math.uni-goettingen.de

M. Wardetzky

e-mail: wardetzky@math.uni-goettingen.de

K. Polthier

Department of Mathematics and Computer Science, Freie Universität Berlin, Arnimallee 6, 14195 Berlin, Germany
} 
Fig. 1 A discrete net of curvature lines on an ellipsoid

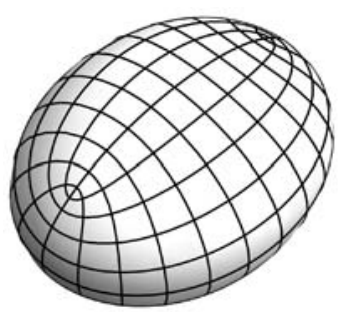

(iii) consider generalizations of integrated, or total, curvatures and yield convergence in the sense of measures or appropriate Sobolev norms, respectively.

Given the convergence of curvatures studied by approaches (ii) and (iii) in an integrated sense, it is natural to ask whether these curvatures can be shown to also converge in a pointwise manner. An affirmative answer can be obtained in some special cases, such as polyhedral surfaces with vertices on the unit 2-sphere [23]. In general, however, the answer to this question is negative: it was observed in [24] that for general irregular polyhedral surfaces, there exist no $k$-local definitions of discrete curvatures that are pointwise convergent. Here, by $k$-locality we mean that the definition of curvatures associated with a vertex $p$ of a polyhedral surface only depends on the $k$-star of $p$, i.e., those vertices that are connected to $p$ by a path of at most $k$ edges. The concept of $k$-locality is motivated by the smooth setting, where the definition of curvatures and differential operators only depends on local properties of the underlying Riemannian manifold.

Uniform Convergence from Nets of Curvature Lines We provide an affirmative answer to the above question of pointwise convergence of curvatures for a special class of discrete meshes: discrete nets of curvature lines on a given smooth surface $M$ that is immersed into Euclidean space $\mathbb{E}^{3}$ (see Fig. 1). To obtain approximations $\left(k_{1}, k_{2}\right)$ of principal curvatures $\left(\kappa_{1}, \kappa_{2}\right)$ on $M$, we follow a three-step approach. We consider (a) local polyhedral approximations to nets of curvature lines, to which we apply (b) well-known 1-local integrated notions of discrete curvatures, such as those based on normal cycles (see, e.g., [8]) or those based on the so-called cotangent formula (see, e.g., [19]), followed by (c) dividing the resulting integrated curvatures by appropriate area terms. The resulting pointwise curvature approximations $k_{1}, k_{2}: V \rightarrow \mathbb{R}$ are at first only defined on the vertex set $V$ of the underlying net. However, we may regard these curvature approximations as functions $k_{i}: M \rightarrow \mathbb{R}$ by extending them from $V$ in a piecewise constant manner to the intrinsic Voronoi regions of the set $V$ on $M$. Assuming this extension, we show:

Theorem 1 Let $M$ be a smooth compact oriented surface without boundary ${ }^{1}$ immersed into $\mathbb{E}^{3}$. Consider a discrete net of curvature lines on $M$ such that at each vertex the sampling condition (10) is satisfied. Let $\epsilon$ be an upper bound for the edge lengths of the net such that additionally the intrinsic $\epsilon$-balls around vertices cover all

\footnotetext{
${ }^{1}$ Surfaces with nonempty boundary can be treated with minor technical modifications.
} 
of $M$. Then

$$
\sup _{p \in M}\left|k_{i}(p)-\kappa_{i}(p)\right| \leq C \epsilon, \quad i=1,2,
$$

where $C$ depends only on properties of $M$ and the shape regularity (9) of the net of curvature lines.

A few remarks seem pertinent before proceeding:

- Uniform pointwise convergence of principal curvatures obtained by a 1-local construction from nets of curvature lines is somewhat surprising since in general 1-local polyhedral curvatures may not even converge in $L^{2}$, even if the mesh vertices reside on the smooth limit surface [15].

- Our pointwise curvature approximations arise from dividing integrated "Steinertype" curvatures by associated area terms. For example, the integrated mean curvature of an interior edge of a polyhedral surface may be defined as the product between the length of that edge and the signed angle between the normals of its adjacent faces-a definition that arises from Steiner's view of considering offset surfaces. To obtain pointwise curvature approximations from discrete integrated curvatures, we divide by so-called circumcentric areas. While this approach is not new, see, e.g., [9], our convergence result may be interpreted as a justification of this construction, provided that the edges of a polyhedral surface well approximate the principal curvature directions of a smooth limit surface. We prove uniform lower and upper bounds for edge-based circumcentric areas that may be of interest in their own right.

- For our result to hold, we require the explicit knowledge of positions of the vertices of a net of curvature lines on a smooth surface and the combinatorics of this net. More precisely, our curvature approximations at a vertex $p$ require the position of $p$ and the positions of its direct neighbors (with respect to the combinatorics of the net). Note that we do not require the knowledge of the entire net, though. (Given such an entire smooth net, it would be trivial to compute the principal curvatures at its vertices.) It would be desirable to drop from our approach the requirement of the exact knowledge of vertex positions of a smooth net of curvature lines. Here, one avenue for further study might be to consider discrete analogues of curvature line nets, so-called principle contact element nets, see [5].

Our uniform convergence result given in Theorem 1 is a consequence of the corresponding local error estimate given in Theorem 2 . This error estimate holds up to and including umbilical points, where singularities in the curvature line pattern arise. Using a refinement sequence for each of the three surfaces shown in Fig. 2, we observed numerically that although the shape regularity of the net may blow up near umbilics, linear convergence with respect to the maximum edge length $\epsilon$ remains valid in these cases. Our experiments also indicate that linear convergence is optimal.

Alternative Approaches An alternative point of departure for establishing pointwise convergence of discrete curvatures is to give up $k$-locality and to allow for $k \rightarrow \infty$ as the mesh refinement increases. In fact, the above-mentioned convergence results 


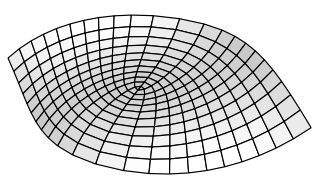

lemon

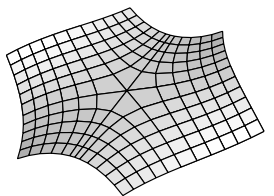

star

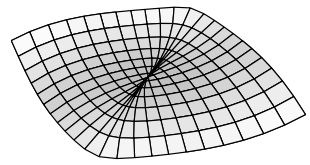

monstar

Fig. 2 The three generic patterns of curvature lines near an umbilical point, called lemon, star, and monstar by Berry and Hannay [2]

of (ii) and (iii) may be interpreted in this way: by decreasing the diameter of the domains over which discrete curvatures are integrated (measured), while simultaneously increasing the mesh refinement inside these domains at a sufficiently fast rate, one recovers classical pointwise notions of smooth curvatures in the limit. In a similar fashion, Belkin et al. [1] proposed a discrete Laplace operator based on the heat kernel. This operator converges in a pointwise manner if the kernel is scaled down while the mesh resolution is increased sufficiently fast relative to the scaling of the kernel. In contrast to these works, which need to allow for $k \rightarrow \infty$ to establish pointwise convergence, our result is obtained by working with the simplest and most local definition: $k=1$.

\section{Discrete Curvatures from Nets of Curvature Lines}

In order to motivate our definition of discrete curvatures for nets of curvature lines, we recall some important notions of curvature for polygonal curves and polyhedral surfaces. For a similar discussion, we refer to [21].

\subsection{Discrete Curvatures of "Steiner-Type"}

Integrated Curvatures for Polygonal Curves Generalizations of classical smooth notions of curvature date at least back to Steiner [20], who considered parallel offsets of convex hypersurfaces, relating integrated or total curvatures to changes in length, area, and enclosed volume. For example, for a convex curve $\gamma \subset \mathbb{E}^{2}$, one of Steiner's formulas reads

$$
l\left(\gamma_{\epsilon}\right)=l(\gamma)+\epsilon \int_{\gamma} \kappa(s) d s,
$$

where $l$ is the length functional, $\kappa$ denotes the curve's curvature, and $\gamma_{\epsilon}$ is the offset curve obtained by displacing $\gamma$ along its normals by some constant amount $\epsilon$.

Steiner's offset formula can be extended to the nonsmooth and nonconvex case $[12,22,25]$. In particular, various notions for curvatures of polygonal curves may be interpreted using Steiner's framework. Consider, e.g.,

$$
k_{p} \in\left\{\theta_{p}, 2 \sin \frac{\theta_{p}}{2}, 2 \tan \frac{\theta_{p}}{2}\right\},
$$



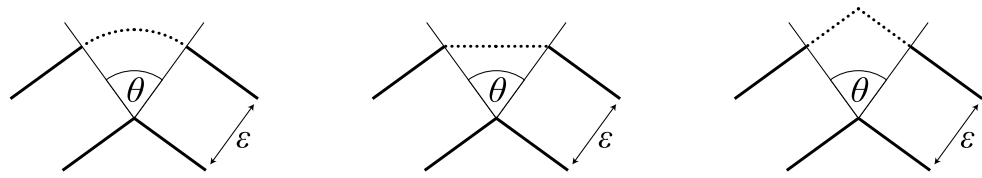

Fig. 3 Applying Steiner's formula (1) to the three depicted definitions of offset curves for a given polygonal curve leads to the three discrete curvatures in (2)

where $p$ denotes an inner vertex of a polygonal curve, and $\theta_{p}$ is the turning angle between the two line segments incident to $p$. These notions arise by applying (1) to the three different types of offsets depicted in Fig. 3. Among these, the first notion is the one considered by Steiner, the second corresponds to a finite element discretization using piecewise linear functions, and the third also arises in the theory of discrete integrable systems $[4,16]$.

Integrated Curvatures for Polyhedral Surfaces By a polyhedral surface, we mean a piecewise linear immersion of a compact simplicial surface into $\mathbb{E}^{3}$. Extending the notions of discrete curvatures from polygonal curves to oriented polyhedral surfaces leads to the following edge-based definitions of integrated normal curvature:

$$
k_{\mathbf{e}} \in\left\{\theta_{\mathbf{e}}\|\mathbf{e}\|, 2 \sin \frac{\theta_{\mathbf{e}}}{2}\|\mathbf{e}\|, 2 \tan \frac{\theta_{\mathbf{e}}}{2}\|\mathbf{e}\|\right\} .
$$

Here $\theta_{\mathbf{e}} \in(-\pi, \pi)$ is the signed angle between the normals of the two flat faces incident to the edge $\mathbf{e}$. Notice that $k_{\mathbf{e}}$ measures curvature orthogonal to $\mathbf{e}$, since there is no curvature along e itself. Integrated mean curvature is accordingly defined as $H_{\mathbf{e}}=\frac{k_{\mathrm{e}}}{2}$.

In the planar limit $\left(\theta_{\mathbf{e}} \rightarrow 0\right)$, the definitions in (3) agree up to second order in the angle variable. Therefore, as it turns out, it suffices to prove convergence of one of these definitions in order to obtain convergence for all of them. Convergence of the first definition in (3) in the sense of measures was investigated in [8, 13].

For completeness, we remark that the above edge-based definitions give rise to vertex-based notions of integrated mean curvatures by adding the mean curvatures over all edges emanating from a given vertex $p$, i.e.,

$$
H_{p}=\frac{1}{2} \sum_{\mathbf{e} \sim p} H_{\mathbf{e}} .
$$

The factor $\frac{1}{2}$ takes the meaning of distributing the normal curvature of each edge equally among its two adjacent vertices.

Finally, the scalar-valued definitions considered so far can be extended to corresponding vector-valued notions. In the edge-based case, we obtain normal curvature vectors $\mathbf{k}_{\mathbf{e}}$ by multiplying $k_{\mathbf{e}}$ with the angle-bisecting unit normal vector at $\mathbf{e}$ (see Fig. 4, left), and similarly for mean curvatures. Analogously to (4), we then obtain vertex-based mean curvature vectors. We remark that for $k_{\mathbf{e}}=2 \sin \frac{\theta_{\mathbf{e}}}{2}\|\mathbf{e}\|$, the resulting mean curvature vector coincides with the surface area gradient at $p$ when restricting to piecewise linear surface variations (yielding the so-called cotangent formula, see [19]). Its convergence in the sense of Sobolev norms was studied in [15]. 

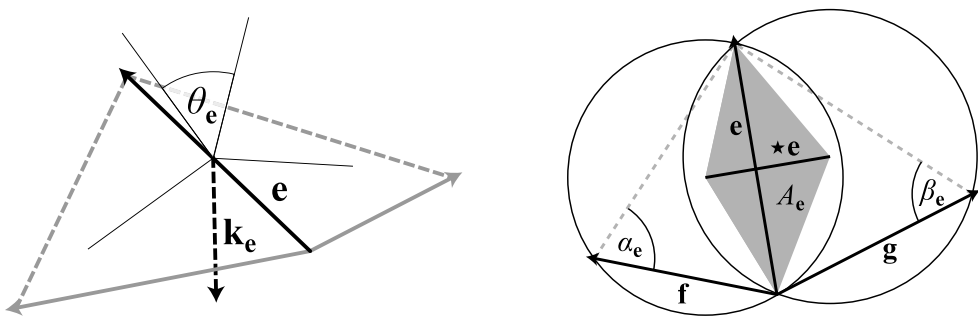

Fig. 4 Edge-based quantities. Left: dihedral angle $\theta_{\mathbf{e}}$ and discrete curvature vector $k_{\mathbf{e}}$. Right: dual edge $\star \mathbf{e}$ and circumcentric area $A_{\mathbf{e}}$

From Integrated to Pointwise Curvatures In order to obtain pointwise curvatures, we divide the above integrated curvatures by corresponding area terms. Intuitively, these areas can be thought of as the domain of integration from which integrated curvatures were obtained. Whether or not one obtains convergent curvatures depends on a careful choice of these areas. It turns out that for triangulated polyhedral surfaces, one good choice are the so-called circumcentric areas, such as considered in [9]. For each edge $\mathbf{e}$, we define

$$
A_{\mathbf{e}}=\frac{1}{2} \operatorname{sgn}(\star e)\|\mathbf{e}\|\|\star \mathbf{e}\|,
$$

where $\|\star \mathbf{e}\|$ denotes the intrinsic length of the circumcentric dual edge $\star \mathbf{e}$. This dual edge intrinsically connects the circumcenters $C_{1}$ and $C_{2}$ of the two triangles $T_{1}$ and $T_{2}$ incident to e. Here, intrinsic means that one can think of $T_{1}$ and $T_{2}$ as being unfolded onto the plane (see Fig. 4, right). The sign $\operatorname{sgn}(\star \mathbf{e})$ is positive if along the direction of the ray from $C_{1}$ through $C_{2}$, triangle $T_{1}$ lies before $T_{2}$, and negative otherwise. Note that $\operatorname{sgn}(\star \mathbf{e}) \leq 0$ (and therefore $A_{\mathbf{e}} \leq 0$ ) iff $\alpha_{\mathbf{e}}+\beta_{\mathbf{e}} \geq \pi$, where $\alpha_{\mathbf{e}}$ and $\beta_{\mathbf{e}}$ are the angles opposite to $\mathbf{e}$ in the triangulation (see Fig. 4, right). Consequently, we require lower bounds that ensure positivity of circumcentric areas. For nets of curvature lines, we provide such bounds in Sect. 3.1.

Similar to vertex-based integrated curvatures, we obtain vertex-based circumcentric areas from the edge-based case via

$$
A_{p}=\frac{1}{2} \sum_{\mathbf{e} \sim p} A_{\mathbf{e}},
$$

where the sum is taken over all edges emanating from a given vertex $p$. If all edges e incident to a vertex $p$ are intrinsically Delaunay (compare [3]), then $A_{p}$ coincides with the intrinsic Voronoi area of $p$ and is therefore positive. However, as pointed out in [10], $A_{p}$ might become negative in general, and a bound similar to the edge-based case is not possible. Therefore, we will not treat vertex-based pointwise curvatures based on $A_{p}$.

\subsection{A Local Error Estimate for Discrete Curvatures}

In this section, we state our main local error estimate (Theorem 2), from which we derive our global uniform convergence result (Theorem 1). 
Fig. 5 The triangulated vertex star at a vertex $p$ is a polyhedral surface approximating the (curved) discrete net of curvature lines at $p$

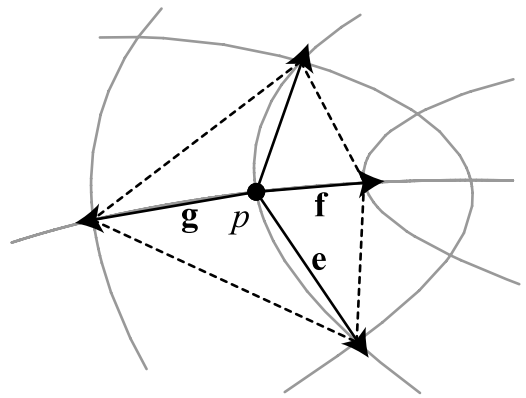

Throughout we assume that $M$ is a smooth compact oriented surface without boundary immersed into $\mathbb{E}^{3}$. By a discrete net on $M$ we mean a cellular decomposition of $M$ such that all attaching maps are homeomorphisms and the intersection of any two cells is either empty or a single cell. As usual, we denote by $E$ the set of edges and by $V$ the set of vertices. We also assume that all edges are smoothly embedded. In a discrete net of curvature lines on $M$, all edges are additionally required to be segments of curvature lines, nonumbilical vertices are required to have valence four, and umbilical vertices are required to have valence greater than two. In a completely umbilical region ( such as $\mathbb{S}^{2}$ ), any net in the above sense serves as a net of curvature lines for our purposes.

In order to be able to apply the concepts of discrete curvatures on polyhedral surfaces to nets of curvature lines, we require local polyhedral approximations of smooth curvature line nets.

Local Polyhedral Approximation In the sequel, the letters e, $\mathrm{f}$, and $\mathrm{g}$ will be reserved for (curved) edges in the edge set $E$, incident to a common vertex $p$, while the corresponding bold face letters, $\mathbf{e}, \mathbf{f}$, and $\mathbf{g}$, will denote the straight edge vectors in $\mathbb{E}^{3}$ obtained by connecting the endpoints of $e, f$, and $g$, respectively, by straight lines (see Fig. 5). We additionally assume that these edge vectors are oriented so that they point away from $p$. For each disjoint edge pair $(e, f)$ incident to $p$ and contained in a common 2-cell, we consider the flat triangle spanned by $\mathbf{e}$ and $\mathbf{f}$. The union of these triangles forms the triangulated vertex star of $p$, denoted by $\operatorname{st}(p)$. Whenever we consider the triple $(\mathbf{e}, \mathbf{f}, \mathbf{g})$, we will always assume that the pairs $(\mathbf{e}, \mathbf{f})$ and $(\mathbf{e}, \mathbf{g})$ span two triangles in $s t(p)$, so that $\mathbf{e}$ is their common edge. Finally, as later justified by our sampling condition (10) and Corollary 9, we may assume that $\mathbf{n} \cdot(\mathbf{e} \times \mathbf{f})>0$ and $\mathbf{n} \cdot(\mathbf{e} \times \mathbf{g})<0$, where $\mathbf{n}$ denotes the normal of $M$ at $p$.

Each triangulated vertex star $s t(p)$ thus yields the requisite local polyhedral approximation, ${ }^{2}$ which forms the basis for our curvature approximations. As outlined in the previous section, our definition of pointwise curvatures relies on the division by certain circumcentric areas, which may become zero or negative in general. This motivates, for a given principal direction, to choose the associated edge vector with maximal circumcentric area.

\footnotetext{
${ }^{2}$ Observe that we do not require that our local polyhedral approximations yield a consistent global one.
} 
Fig. 6 A discrete net of curvature lines, with the two families of directions depicted as solid and dotted lines, respectively, together with the circumcentric areas (gray and white diamonds) per edge

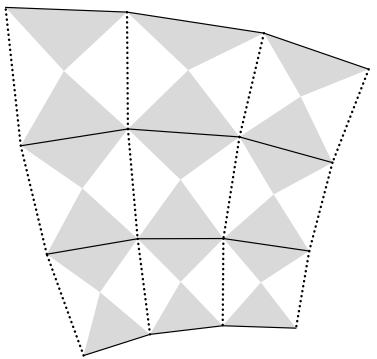

Definition 1 (Area maximizing edge) Consider a vertex $p$ in a discrete net of curvature lines. If $p$ is umbilical, we call an edge vector e area maximizing if it maximizes the circumcentric area among all edges emanating from $p$ in the local polyhedral approximation. If $p$ is nonumbilical, let $\mathbf{v}_{1}$ be the principal direction canonically associated with an edge vector $\mathbf{e}$. We call $\mathbf{e}$ area maximizing if it maximizes the circumcentric area among the two edge vectors associated with $\mathbf{v}_{1}$.

We show in Sect. 3.1 that area maximizing edges always have circumcentric areas that are bounded away from zero.

Definition 2 (Principal curvature approximations) Consider a vertex $p$ in a discrete net of curvature lines and let $\mathbf{e}$ be an area maximizing edge (associated with a principal direction $\mathbf{v}_{1}$ if $p$ is nonumbilical). Then

$$
k_{2}(p):=\frac{k_{\mathbf{e}}}{2 A_{\mathbf{e}}}
$$

defines the principal curvature approximation of $\kappa_{2}(p)$, where $\kappa_{2}$ refers to the principal curvature corresponding to $\mathbf{v}_{2}$ if $p$ is nonumbilical and refers to the unique normal curvature if $p$ is umbilical. Here $k_{\mathbf{e}}$ is one of the edge-based integrated polyhedral curvatures defined in (3).

The fact that in the above definition the edge vector $\mathbf{e}$ is associated with $\mathbf{v}_{1}$ while $\frac{k_{\mathbf{e}}}{2 A_{\mathbf{e}}}$ approximates $\kappa_{2}$ is not an oversight: $k_{\mathbf{e}}$ measures curvature orthogonal to e.

The intuitive reason for dividing by twice the circumcentric area in the above definition may (at least qualitatively) be explained as follows. Consider Fig. 6, where one set of principal curvature directions, say those associated with $\mathbf{v}_{1}$, is depicted by solid lines, while the direction corresponding to $\mathbf{v}_{2}$ is represented by dotted ones. Likewise, the circumcentric areas corresponding to $\mathbf{v}_{1}$-directions are drawn as gray diamonds, while the circumcentric areas corresponding to $\mathbf{v}_{2}$-directions are represented as white diamonds. Roughly, the gray diamonds cover only half of the total surface. Hence, taking only the gray diamonds as regions of support for our (integrated) principal curvature approximations corresponding to $\kappa_{1}$, would mean to be roughly missing a factor of two. This motivates, for each edge along a given principal direction, to consider twice its circumcentric area as the domain of integration. 
Global Constants For each $p \in M$, let $S(p)$ denote the shape (or Weingarten) operator. Our estimates depend on both $S$ and its covariant derivative, $\nabla S$. Accordingly, we define

$$
\mathcal{K}:=\max _{p}\|S(p)\|_{\text {op }} \quad \text { and } \quad \mathcal{K}^{\prime}:=\max _{p}\left(\max _{\|\mathbf{v}\|=1}\left\|\nabla_{\mathbf{v}} S(p)\right\|_{\text {op }}\right),
$$

where $\|\cdot\|_{\text {op }}$ denotes the usual norm for linear operators. Note that $\mathcal{K}$ is an upper bound for the normal curvatures of $M$, whereas $\mathcal{K}^{\prime}$ provides an upper bound for directional derivatives $\nabla_{\mathbf{v}}\left(\kappa_{i}\right)$ of principal curvatures $\kappa_{i}$.

Local Constants We also consider local constants - shape regularity $\rho$ and maximum edge length $\epsilon$-that are specific for each vertex in the net of curvature lines. The reason for introducing local constants is that a high aspect ratio at one vertex should not affect the sampling condition (see below) at another vertex. In the following, we assume an arbitrary but fixed vertex $p$.

We let $\epsilon$ denote the largest intrinsic edge length over all (curved) edges $e \in E$ emanating from $p \in V$ (denoted by $e \sim p$ ),

$$
\epsilon=\max _{e \sim p} l(e) .
$$

Notice that the length of every edge vector emanating from $p$ in $s t(p)$ is thus also bounded above by $\epsilon$.

Our estimates also depend on shape regularity, or aspect ratio. We define $\rho \geq 1$ to be the smallest number such that for all pairs $(\mathbf{e}, \mathbf{f})$ of edge vectors emanating from $p$ and forming a triangle in $s t(p)$, one has

$$
\frac{\epsilon}{\rho} \leq\|\mathbf{e}\| \quad \text { and } \quad \frac{\|\mathbf{e}\|\|\mathbf{f}\|}{\|\mathbf{e} \times \mathbf{f}\|} \leq \rho .
$$

The former inequality implies $\frac{1}{\rho} \leq \frac{\|\mathbf{e}\|}{\|\mathbf{f}\|} \leq \rho$, while the latter means $\sin \angle(\mathbf{e}, \mathbf{f}) \geq \frac{1}{\rho}$.

Sampling Condition In addition to the above definitions of maximum (local) edge length and (local) shape regularity, we assume the (local) sampling condition

$$
\epsilon \leq \frac{1}{16 \mathcal{K} \rho^{2}}
$$

In some of our estimates, it will suffice to work with weaker sampling conditions, such as $\epsilon \leq \frac{1}{2 \mathcal{K}_{\rho}}$ or $\epsilon \leq \frac{1}{2 \mathcal{K}}$, both of which are implied by (10).

Theorem 2 (Local error estimate) Let $M$ be a smooth compact oriented surface without boundary immersed into $\mathbb{E}^{3}$. Consider a vertex $p \in V$ in a discrete net of curvature lines on $M$ and assume the sampling condition (10). Let $\left(k_{1}(p), k_{2}(p)\right)$ denote the approximations of the smooth principal curvature $\left(\kappa_{1}(p), \kappa_{2}(p)\right)$ as in Definition 2. Then

$$
\left|k_{i}(p)-\kappa_{i}(p)\right| \leq C \epsilon, \quad i=1,2 .
$$


The constant $C=C\left(\mathcal{K}, \mathcal{K}^{\prime}, \rho\right)$ depends only on the curvature bounds (7) and the shape regularity (9).

Remark Equivalent estimates can be obtained when replacing the scalar-valued quantities in (11) by their corresponding vector-valued counterparts (for definitions, see Sect. 2). Proofs remain nearly identical.

Our global uniform convergence theorem stated in the introduction is a direct consequence of our local error estimate.

Proof of Theorem 1 Let $f: M \rightarrow V$ be defined by mapping each $p \in M$ to its nearest point in the vertex set $V$, i.e., $d_{M}(p, f(p))=d_{M}(p, V)$, where $d_{M}$ denotes the intrinsic distance on $M$. We extend our curvature approximations (initially only defined on $V$ ) to functions $k_{i}: M \rightarrow \mathbb{R}$ via $p \mapsto k_{i}(f(p))$. By the assumptions of Theorem 1 , $\epsilon$ was globally chosen such that $d_{M}(p, f(p)) \leq \epsilon$ for all $p \in M$. This implies

$$
\left|\kappa_{i}(p)-\kappa_{i}(f(p))\right| \leq \mathcal{K}^{\prime} \epsilon
$$

Furthermore, $\epsilon$ was globally chosen such that it maximizes global edge length. Hence Theorem 2 implies that there exists a constant $\tilde{C}$ such that

$$
\left|k_{i}(f(p))-\kappa_{i}(f(p))\right| \leq \tilde{C} \epsilon
$$

for all $p \in M$. Additionally, note that the constant $C$ in Theorem 2, besides depending on $\mathcal{K}$ and $\mathcal{K}^{\prime}$, is monotonically increasing with respect to shape regularity. (This will become evident in the proof of Theorem 2.) Hence, $\tilde{C}$ depends only on $\mathcal{K}, \mathcal{K}^{\prime}$, and the largest local shape regularity constant $\rho$. This, together with an application of the triangle inequality,

$$
\begin{aligned}
\left|k_{i}(p)-\kappa_{i}(p)\right| & =\left|k_{i}(f(p))-\kappa_{i}(p)\right| \\
& \leq\left|k_{i}(f(p))-\kappa_{i}(f(p))\right|+\left|\kappa_{i}(f(p))-\kappa_{i}(p)\right|,
\end{aligned}
$$

implies the claim.

\section{Proof of Local Error Estimate}

The proof of Theorem 2 proceeds in several steps. First, we provide uniform lower and upper bounds for the edge-based circumcentric areas by which we divide integrated curvatures to obtain pointwise notions (Sect. 3.1). In a second step, we provide estimates for edge-based integrated curvatures by using their corresponding discrete curvature vector. We establish that for each vertex $p$ in a net of curvature lines, the projection of these vectors onto the tangent plane $T_{p} M$ is negligible. Furthermore, we show that the remaining normal component leads to the error estimate in Theorem 2 up to a certain error term (Sect. 3.2). While for general meshes, the resulting error term cannot be controlled (and indeed causes failure of pointwise convergence), 
we provide bounds for this error term for the specific case of nets of curvature lines (Sect. 3.3).

Basic Assumptions In order to avoid excessive repetition, we summarize our basic assumptions and notation. We write $p$ for a nonboundary vertex of a polyhedral surface, with vertex star denoted by $s t(p)$. We assume that $\epsilon$ is an upper bound for the length of the (straight) edges emanating from $p$, and we let $\rho$ denote the shape regularity as defined in (9). If $s t(p)$ arises from the local polyhedral approximation of a net of curvature lines, then $\epsilon$ is defined by (8), i.e., as the maximum edge length of the curved edges emanating from $p$. Throughout, we assume the sampling condition (10). As before, we denote curved edges by $e, f$, and $g$, and their corresponding straight edge vectors by $\mathbf{e}, \mathbf{f}$, and $\mathbf{g}$.

\subsection{Uniform Bounds for Circumcentric Areas}

In this section we prove upper and lower bounds for the circumcentric areas of area maximizing edges in the sense of Definition 1.

Proposition 3 Consider a vertex $p$ in a discrete net of curvature lines and let $\mathbf{e}$ be an area maximizing edge vector at $p$. Then our basic assumptions imply the existence of some $C>0$ such that

$$
\frac{1}{C} \epsilon^{2} \leq A_{\mathbf{e}} \leq C \epsilon^{2}
$$

where $C$ only depends on the shape regularity constant $\rho$.

Note that for nonumbilical vertices, this result implies the existence of an edge with positive circumcentric area for each of the two principal curvature directions.

The remainder of this section is concerned with proving Proposition 3. First observe that

$$
A_{\mathbf{e}}=\frac{1}{4}\left(\cot \alpha_{\mathbf{e}}+\cot \beta_{\mathbf{e}}\right)\|\mathbf{e}\|^{2}=\frac{\sin \left(\alpha_{\mathbf{e}}+\beta_{\mathbf{e}}\right)}{4 \sin \alpha_{\mathbf{e}} \sin \beta_{\mathbf{e}}}\|\mathbf{e}\|^{2},
$$

where $\alpha_{\mathbf{e}}$ and $\beta_{\mathbf{e}}$ are the angles opposing $\mathbf{e}$ in the two triangles meeting at $\mathbf{e}$, respectively. The requisite upper bound on $A_{\mathbf{e}}$ is relatively straightforward to obtain.

Lemma 4 (Upper bound) Let $p$ be a vertex in a discrete net of curvature lines on $M$. Then our basic assumptions imply that each edge e emanating from $p$ satisfies

$$
A_{\mathbf{e}} \leq \rho^{4} \epsilon^{2} \text {. }
$$

Proof Clearly, we have

$$
A_{\mathbf{e}} \leq \frac{\|\mathbf{e}\|^{2}}{4 \sin \alpha_{\mathbf{e}} \sin \beta_{\mathbf{e}}}
$$


Let $\mathbf{f}$ be the edge vector emanating from $p$ such that $\alpha_{\mathbf{e}}$ belongs to the triangle formed by $\mathbf{e}$ and $\mathbf{f}$. Then the definition of shape regularity (9) implies

$$
\sin \alpha_{\mathbf{e}}=\frac{\|(\mathbf{f}-\mathbf{e}) \times \mathbf{f}\|}{\|(\mathbf{f}-\mathbf{e})\|\|\mathbf{f}\|} \geq \frac{\|\mathbf{e} \times \mathbf{f}\|}{(\rho+1)\|\mathbf{e}\|\|\mathbf{f}\|} \geq \frac{1}{(\rho+1) \rho} \geq \frac{1}{2 \rho^{2}} .
$$

A similar estimate holds for $\beta_{\mathbf{e}}$. Hence, $A_{\mathbf{e}} \leq \rho^{4}\|\mathbf{e}\|^{2} \leq \rho^{4} \epsilon^{2}$.

Similarly, we obtain a lower bound for at least one edge emanating from $p$.

Lemma 5 (Lower bound) Let $p$ be a vertex in a discrete net of curvature lines on $M$. Then our basic assumptions imply that there exists an edge vector e emanating from $p$ such that

$$
A_{\mathbf{e}} \geq \frac{1}{4 \rho^{3}} \epsilon^{2}
$$

Proof Let $\mathbf{e}$ be the shortest edge emanating from $p$, let $\mathbf{f}$ be the straight edge emanating from $p$ such that $\alpha_{\mathbf{e}}$ belongs to the triangle formed by $\mathbf{e}$ and $\mathbf{f}$, and define $\gamma:=\angle(\mathbf{e}, \mathbf{f})$. Since $\|\mathbf{e}\| \leq\|\mathbf{f}\|$, it follows that $2 \alpha_{\mathbf{e}} \leq(\pi-\gamma)$. Since in particular $0<\alpha_{\mathbf{e}}<\frac{\pi}{2}$, it follows that $\cot \alpha_{\mathbf{e}}>\frac{\pi}{2}-\alpha_{\mathbf{e}}$. By (9) we have $\sin \gamma \geq \frac{1}{\rho}$, and hence

$$
\cot \alpha_{\mathbf{e}}>\frac{\pi}{2}-\alpha_{\mathbf{e}} \geq \frac{\gamma}{2} \geq \frac{\sin \gamma}{2} \geq \frac{1}{2 \rho}
$$

Applying similar arguments, we obtain $\cot \beta_{\mathbf{e}}>\frac{1}{2 \rho}$. Together, this yields

$$
A_{\mathbf{e}}=\frac{1}{4}\left(\cot \alpha_{\mathbf{e}}+\cot \beta_{\mathbf{e}}\right)\|\mathbf{e}\|^{2} \geq \frac{1}{4 \rho}\|\mathbf{e}\|^{2} \geq \frac{1}{4 \rho^{3}} \epsilon^{2} .
$$

Lower Bounds for Vertices of Valence Four The above lower bound on the circumcentric area of at least one edge suffices for umbilical vertices. However, it does not suffice at nonumbilical ones, since we require lower bounds for edges associated with each of the two principal directions. We achieve this by showing that for each vertex of valence four, there are at least three edges that satisfy the required lower bound. Hence, for each vertex in a net of curvature lines, we have at least one good edge per principal direction.

We note that some of the following results (in particular, Corollary 9) are also valid for vertices of valence different from four.

As before, we let $\alpha_{\mathbf{e}}$ and $\beta_{\mathbf{e}}$ denote the angles opposing the straight edge $\mathbf{e}$ in the two triangles meeting at $\mathbf{e}$, respectively. If $\alpha_{\mathbf{e}} \geq \delta, \beta_{\mathbf{e}} \geq \delta$, and $\alpha_{\mathbf{e}}+\beta_{\mathbf{e}} \leq \pi-\delta$ for some $\delta>0$, then

$$
A_{\mathbf{e}} \geq \frac{\sin \delta\|\mathbf{e}\|^{2}}{4},
$$

which provides a useful lower bound if $\delta$ can be bounded away from zero. Accordingly, we introduce the notion of $\delta$-Delaunay edges, a nomenclature that is borrowed from the classical case of Delaunay triangulations (corresponding to $\delta=0$ ). 
Definition 3 ( $\delta$-Delaunay) Let $\alpha_{\mathbf{e}}$ and $\beta_{\mathbf{e}}$ be the angles opposing an edge $\mathbf{e}$ in the two triangles meeting at $\mathbf{e}$, respectively. Then $\mathbf{e}$ is called $\delta$-Delaunay if there exists $\delta \geq 0$ such that $\alpha_{\mathbf{e}} \geq \delta, \beta_{\mathbf{e}} \geq \delta$, and $\alpha_{\mathbf{e}}+\beta_{\mathbf{e}} \leq \pi-\delta$.

Assume for the moment that $p$ has valence four and that $s t(p)$ is planar. Assume further that all of the eight angles opposing the four edges emanating from $p$ are bounded from below by $\delta$. Then it is straightforward to verify that at least three among the four edges incident to $p$ are $\delta$-Delaunay. In general, however, $s t(p)$ is not planar, and we have to account for Gaussian curvature. As usual, we define discrete Gauss curvature at a vertex $p$ of a polyhedral surface as the angle defect, i.e., by $K_{p}=$ $2 \pi-\sum_{i} \gamma_{i}$, where $\gamma_{i}$ are the intrinsic angles meeting at $p$. We obtain:

Lemma 6 Let $p$ be a nonboundary vertex of valence four on a triangulated polyhedral surface, and let $\alpha_{i}, \beta_{i}$ denote the pairs of angles opposing the four edges emanating from $p$. Assume that $\alpha_{i} \geq \delta$ and $\beta_{i} \geq \delta$ for all $i=1, \ldots, 4$ and that $K_{p}<2 \delta$. Then at least three among the four edges meeting at $p$ are $\delta$-Delaunay.

Proof Using $K_{p}=-2 \pi+\sum_{i=1}^{4}\left(\alpha_{i}+\beta_{i}\right)$, the result follows from a straightforward calculation.

We now show that our basic assumptions imply the assumptions of Lemma 6 when setting $\delta:=\frac{1}{2 \rho^{2}}$. To see this, first observe that with the above notation, $\alpha_{\mathbf{e}} \geq \sin \alpha_{\mathbf{e}} \geq \delta$ by (12), and analogously for $\beta_{\mathbf{e}}$. It remains to check the condition $K_{p}<2 \delta$ for the discrete Gauss curvature. The requisite bound will be established in Lemmas 7 and 8 . The resulting consequence for the existence of $\delta$-Delaunay edges is summarized in Lemma 10. Finally, Lemma 11 establishes the lower bound for $A_{\mathbf{e}}$.

Lemma 7 Let $p$ be a nonboundary vertex of an oriented polyhedral surface. Assume that the normals of the triangles incident to $p$ make an angle no greater than $\phi \in$ $[0, \pi)$ with some fixed direction in $\mathbb{E}^{3}$. Then the discrete Gauss curvature associated with $p$ satisfies $K_{p} \leq 2 \pi(1-\cos \phi)$.

Proof The lemma is a consequence of the Gauss-Bonnet theorem. Let $\mathbf{n}_{1}, \mathbf{n}_{2}, \ldots, \mathbf{n}_{l}$ denote the unit normals of the triangles $T_{1}, T_{2}, \ldots, T_{l}$ incident to $p$, ordered according to the orientation of the polyhedral surface. Each $\mathbf{n}_{i}$ represents a point on $\mathbb{S}^{2}$. Connecting consecutive pairs $\left(\mathbf{n}_{i}, \mathbf{n}_{i+1}\right)$ by geodesic arcs on $\mathbb{S}^{2}$ yields a spherical polygon $P$ (possibly with intersecting edges). For each $i$, the exterior angle of $P$ at $\mathbf{n}_{i}$ is then equal to the interior angle of the Euclidean triangle $T_{i}$ at $p$. In particular, the sum $\Sigma$ of the exterior angles of $P$ satisfies $\Sigma=2 \pi-K_{p}$.

Moreover, by assumption we have $\phi \in[0, \pi)$, so all $\mathbf{n}_{i}$ lie on the same hemisphere. We can hence consider the spherical convex hull $\tilde{P}$ of $\mathbf{n}_{1}, \mathbf{n}_{2}, \ldots, \mathbf{n}_{l}$, i.e., the smallest spherical polygon that contains all $\mathbf{n}_{i}$ and that is convex with respect to shortest geodesic arcs. Let $\tilde{\Sigma}$ denote the sum of exterior angles of $\tilde{P}$. It is easy to verify that $\tilde{\Sigma} \leq \Sigma$. Hence $K_{p} \leq 2 \pi-\tilde{\Sigma}=\operatorname{area}(\tilde{P})$, where the last equality follows from the Gauss-Bonnet theorem. Since $\phi \in[0, \pi)$, the polygon $\tilde{P}$ is contained in a geodesic disk of radius $\phi$, the area of which is $2 \pi(1-\cos \phi)$. This proves the claim. 
In order to make use of the previous lemma, we seek a bound on the angle $\phi$ between the surface normal at $p \in M$ and the normals to the triangles incident to $p$. Note that our basic assumptions, and in particular our sampling condition, imply $\epsilon \leq \frac{1}{2 \mathcal{K}}$, where $\mathcal{K}$ is our curvature bound. Hence we can infer from [18, Sect. 3 , Corollary 1]:

Lemma 8 Let $p$ be a vertex (umbilical or not) in a net of curvature lines on $M$. Given our basic assumptions, st $(p)$ can be oriented so that the maximum angle $\phi \in$ $[0, \pi)$ between the surface normal at $p \in M$ and the normals to the triangles in $s t(p)$ satisfies $\sin \phi \leq(4 \rho+2) \mathcal{K} \epsilon$, where $\rho$ is the shape regularity. In particular, $\sin \phi \leq \frac{3}{8}$.

Corollary 9 Under the assumptions of Lemma 8, st $(p)$ can be oriented so that the orthogonal projection of $s t(p)$ onto $T_{p} M$ is injective and orientation-preserving.

Note that our assumptions are slightly different from those used in [18]; in fact, our assumptions are stricter. While their sampling condition bounds the maximal extrinsic distance between two neighboring vertices, we consider the intrinsic length on the smooth surface, which is always larger. Additionally, in [18] it is assumed that the distance between the discrete and the smooth surface is less than the reach of the smooth one. This assumeption is implicitly fulfilled locally by the sampling condition $\epsilon \leq \frac{1}{2 \mathcal{K}}$, since the reach of a surface patch formed by an intrinsic $\epsilon$-disk around $p$ is nothing but the minimal radius of curvature of that surface patch.

Lemma 10 In addition to the assumptions of Lemma 8, assume that $p$ is of valence four. Let $\delta:=\frac{1}{2 \rho^{2}}$, where $\rho$ is the shape regularity. Then at least three edges incident to $p$ are $\delta$-Delaunay.

Proof Using that $(1-\cos \phi) \leq \sin ^{2} \phi$ for $\phi \in\left[0, \frac{\pi}{2}\right]$, Lemmas 7 and 8 show that the discrete Gauss curvature at $p$ satisfies

$$
K_{p} \leq 2 \pi(4 \rho+2)^{2} \mathcal{K}^{2} \epsilon^{2} \leq(16 \mathcal{K} \rho \epsilon)^{2} .
$$

Setting $\delta=\frac{1}{2 \rho^{2}}$, the sampling condition (10) implies $K_{p} \leq 2 \delta$. Moreover, (12) implies $\alpha_{i} \geq \delta$ and $\beta_{i} \geq \delta$ for all pairs of angles $\alpha_{i}, \beta_{i}$ opposing the four straight edges emanating from $p$. Finally, Lemma 6 implies the claim.

Lemma 11 (Lower bound for valence four) Under the assumptions of Lemma 10, there exist at least three straight edges among the four edges emanating from $p$ such that

$$
A_{\mathbf{e}} \geq \frac{1}{16 \rho^{4}} \epsilon^{2}
$$

for each edge e among these three. 
Proof Observe that (13) implies $4 A_{\mathbf{e}} \geq \sin \delta\|\mathbf{e}\|^{2}$ for all $\delta$-Delaunay edges. Applying Lemma 10 and using $\sin \left(\frac{1}{2 \rho^{2}}\right) \geq \frac{1}{4 \rho^{2}}$ gives

$$
A_{\mathbf{e}} \geq \frac{1}{4} \sin \left(\frac{1}{2 \rho^{2}}\right)\|\mathbf{e}\|^{2} \geq \frac{\|\mathbf{e}\|^{2}}{16 \rho^{2}} \geq \frac{1}{16 \rho^{4}} \epsilon^{2} .
$$

Proof of Proposition 3 Lemma 4 provides an upper bound for both umbilical and nonumbilical vertices. Lemma 5 provides the requisite lower bound for umbilical vertices. Finally, Lemma 11 provides the lower bound for nonumbilical ones, since it implies the existence of at least one edge per principal direction with circumcentric area bounded from below.

\subsection{Estimates for Discrete Integrated Curvatures}

In this section, we establish a bound for the difference between the edge-based integrated curvatures $k_{\mathbf{e}}$ and (an appropriately scaled version of) the smooth principal curvatures of $M$. Specifically, for a given edge $\mathbf{e}$ in our polyhedral approximation, we work with the discrete integrated curvature $k_{\mathbf{e}}=2 \sin \frac{\theta_{\mathbf{e}}}{2}\|\mathbf{e}\|$ introduced in Sect. 2.1.

Darboux Frames For our purposes, it turns out to be useful to express vectors in frames that are locally adapted to the geometry of the surface $M$. Specifically, a Darboux frame at a nonumbilical point $p \in M$ is an adapted frame given by $\left(\mathbf{v}_{1}, \mathbf{v}_{2}, \mathbf{n}\right)$, where $\mathbf{v}_{1}$ and $\mathbf{v}_{2}$ are (normalized) principal directions of $M$ at $p$, and $\mathbf{n}=\mathbf{v}_{1} \times \mathbf{v}_{2}$ is the surface normal (induced by the orientation of $M$ ). For umbilical points, any adapted (i.e., $\mathbf{v}_{1}, \mathbf{v}_{2} \in T_{p} M$ ) and orthonormal (i.e., $\left\|\mathbf{v}_{1}\right\|=\left\|\mathbf{v}_{2}\right\|=1$ and $\mathbf{n}=\mathbf{v}_{1} \times \mathbf{v}_{2}$ ) frame may be considered a Darboux frame. Throughout, we employ the notation

$$
\mathbf{e}=\left(e_{1}, e_{2}, e_{n}\right)
$$

to represent a vector $\mathbf{e}$ in the coordinates given by a Darboux frame. In the sequel, we assume a fixed Darboux frame at every vertex $p$ of our discrete net of curvature lines. If $p$ is nonumbilical, then each edge vector emanating from $p$ is canonically associated to exactly one of the principal directions $\mathbf{v}_{1}$ or $\mathbf{v}_{2}$. If $p$ is umbilical, we additionally require an explicit association of each edge vector with one of either $\mathbf{v}_{1}$ or $\mathbf{v}_{2}$. In order to state the main result of this section, we require the notion of tangential deviation of an edge vector with respect to a Darboux frame.

Definition 4 (Tangential deviation) Let $p$ be a vertex in a discrete net of curvature lines on $M$ and let $\left(\mathbf{v}_{1}, \mathbf{v}_{2}, \mathbf{n}\right)$ be the Darboux frame at $p$. If an edge vector $\mathbf{e}$ is associated with the principal direction $\mathbf{v}_{1}$, we call $e_{d}=\left|e_{2}\right|$ its tangential deviation, see Fig. 7. Likewise, if $\mathbf{e}$ is associated with $\mathbf{v}_{2}$, we let $e_{d}=\left|e_{1}\right|$. 
Fig. 7 The tangential deviation $e_{d}$ of a straight edge $\mathbf{e}$

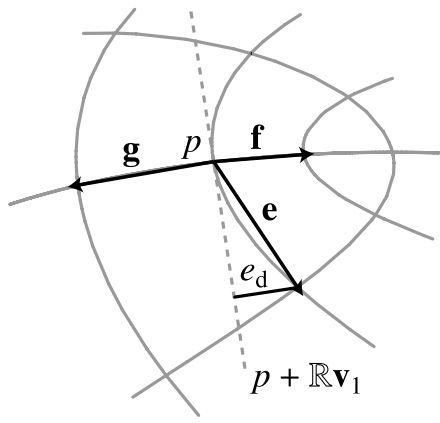

The following proposition summarizes the main result of this section.

Proposition 12 Using the notation of Definition 4, let the edge vector e emanating from a vertex $p$ be associated with $\mathbf{v}_{1}$, and let its directly neighboring edges $\mathbf{f}$ and $\mathbf{g}$ be associated with $\mathbf{v}_{2}$. Then our basic assumptions imply that

$$
\left|k_{\mathbf{e}}-2 A_{\mathbf{e}} \kappa_{2}\right| \leq C\left(\left|\delta_{\kappa}\left(e_{d}+f_{d}+g_{d}\right)\right| \epsilon+\epsilon^{3}\right),
$$

where $\kappa_{2}$ denotes the principal curvature of $M$ in direction $\mathbf{v}_{2}, \delta_{\kappa}=\kappa_{2}-\kappa_{1}$ (with $\delta_{\kappa}=0$ in the umbilical case $)$, and $C=C\left(\mathcal{K}, \mathcal{K}^{\prime}, \rho\right)$.

Remark We point out that the estimates in this section are not entirely specific for nets of curvature lines. In fact, they hold in the more general setting of arbitrary smooth nets embedded in $M$, as long as we require bounded shape regularity and the sampling condition $\epsilon \leq \frac{1}{2 \mathcal{K}}$, where $\epsilon$ is the maximum intrinsic edge length of the net and $\mathcal{K}$ denotes our usual curvature bound. As a consequence, the estimates of the current section do not suffice to establish our main error estimate. Indeed, to obtain uniform convergence, we require that the term $\delta_{\kappa}\left(e_{d}+f_{d}+g_{d}\right)$ appearing in Proposition 12 is of order $\epsilon^{2}$. However, this is false for general nets (and causes failure of uniform convergence) but is true for nets of curvature lines as we will show in Sect. 3.3. For clarity, though, we have decided to restrict the discussion of the current section to nets of curvature lines and rely on our (rather strong) basic assumptions set forth in the beginning of Sect. 3 .

Proposition 12 is proven in several steps. We commence by estimating the normal component $e_{n}$ of the straight edge vector e (Lemma 13 and Corollary 14). Then we turn to the edge-based curvature vector $\mathbf{k}_{\mathbf{e}}$ corresponding to $k_{\mathbf{e}}$. We show that its tangential component is negligible (Lemma 15). The final proof of Proposition 12 is given at the end of this section, where we show that the normal component of $\mathbf{k}_{\mathbf{e}}$ yields the desired estimate.

Lemma 13 Let $p$ be a vertex of a discrete net of curvature lines on M. Consider an edge $e \in E$ emanating from $p$ with corresponding straight edge vector $\mathbf{e}$. Writing $\mathbf{e}=\left(e_{1}, e_{2}, e_{n}\right)$ with respect to a Darboux frame centered at $p$, our basic assumptions 
imply that

$$
\left|e_{n}\right| \leq \mathcal{K} \mathbf{e}^{2} \leq \mathcal{K} \epsilon^{2}
$$

Furthermore, if $P(x, y)=\frac{\kappa_{1}}{2} x^{2}+\frac{\kappa_{2}}{2} y^{2}$ denotes the osculating paraboloid, then

$$
\left|e_{n}-P\left(e_{1}, e_{2}\right)\right| \leq C \epsilon^{3}
$$

where $C$ only depends on our global curvature bound $\mathcal{K}$ and the bound on curvature derivatives $\mathcal{K}^{\prime}$.

Proof Using a Darboux frame at $p$, the surface $M$ can locally be parameterized by a height function $h(x, y)$ over the tangent plane $T_{p} M$. In the coordinates of the Darboux frame, we have $\mathbf{e}=\left(e_{1}, e_{2}, e_{n}\right)$, with $e_{n}=h\left(e_{1}, e_{2}\right)$. We let $d=\left\|\left(e_{1}, e_{2}\right)\right\|$, where throughout this proof $\|\cdot\|$ denotes the Euclidean norm in the parameter domain. Furthermore, we consider the constant vector field $\mathbf{v}(x, y)=\left(e_{1}, e_{2}\right) / d$ in the parameter domain.

Let $D_{\mathbf{v}}^{i} h$ denote the $i$ th iteration of the directional derivative of $h$ along $\mathbf{v}$, i.e., $D_{\mathbf{v}}^{1} h=D_{\mathbf{v}} h$ and $D_{\mathbf{v}}^{i}=D_{\mathbf{v}}\left(D_{\mathbf{v}}^{i-1} h\right)$, and let $D^{i} h$ denote the $i$ th total derivative with respect to the standard Euclidean metric in the parameter domain. Observe that $h(0)=D h(0)=0$, and hence

$$
e_{n}=h\left(e_{1}, e_{2}\right)=\int_{0}^{d} \int_{0}^{t} D_{\mathbf{v}}^{2} h(\tau \mathbf{v}) d \tau d t .
$$

Consequently, in order to prove the first part of the lemma, we seek an upper bound on $\left|D_{\mathbf{v}}^{2} h\right|$ in terms of $\mathcal{K}$.

Let $S$ denote the shape operator, and let $\mathrm{I}(\cdot, \cdot)$ and $\mathrm{I}(\cdot, \cdot)=\mathrm{I}(S \cdot, \cdot)$ be the first and second fundamental forms of $M$, respectively, with respect to the local parameterization induced by $h$. From

$$
\mathrm{I}(\mathbf{u}, \mathbf{v})=\mathbf{u}^{T}\left(\mathrm{Id}+D h D h^{T}\right) \mathbf{v}
$$

and

$$
\kappa_{\mathbf{v}}=\frac{I(\mathbf{v}, \mathbf{v})}{\mathrm{I}(\mathbf{v}, \mathbf{v})}=\frac{\mathrm{I}(S \mathbf{v}, \mathbf{v})}{\mathrm{I}(\mathbf{v}, \mathbf{v})}=\frac{D_{\mathbf{v}}^{2} h}{\sqrt{1+\|D h\|^{2}} \mathrm{I}(\mathbf{v}, \mathbf{v})}
$$

we obtain the estimate

$$
\left|D_{\mathbf{v}}^{2} h\right|=\left|\kappa_{\mathbf{v}}\right| \sqrt{1+\|D h\|^{2}} \mathrm{I}(\mathbf{v}, \mathbf{v}) \leq \mathcal{K}\left(1+\|D h\|^{2}\right)^{\frac{3}{2}} .
$$

In order to bound $\|D h\|$, first observe that our sampling condition implies $\epsilon \leq \frac{1}{2 \mathcal{K}}$. This, in turn, provides a bound on the (positive) angle between the surface normal $\mathbf{n}_{p}$ at $p$ and the surface normal $\mathbf{n}_{q}$ at any point $q$ on the (curved) edge $e \subset M$ incident to $p$ in the given net of curvature lines on $M$. To see this, consider an arc-length parameterized curve $\gamma:[0, \xi] \rightarrow M$ with $\gamma(0)=p$ and $\gamma(\xi)=q$. Notice that our basic assumptions imply that $\gamma$ can be chosen such that $\xi \leq \epsilon$. The Gauss image 
$\tilde{\gamma}=\mathbf{n} \circ \gamma$ of $\gamma$ is a curve on the unit sphere. The length of $\tilde{\gamma}$ is therefore bounded from below by $\angle\left(\mathbf{n}_{p}, \mathbf{n}_{q}\right)$, i.e., the length of the minimizing geodesic joining $\mathbf{n}_{p}$ and $\mathbf{n}_{q}$ on $\mathbb{S}^{2}$. The tangent vector of $\tilde{\gamma}$ at a point $\tilde{\gamma}(s), s \in[0, \xi]$, is given by $S \gamma^{\prime}(s)$, and the norm of this vector is therefore bounded above by $\mathcal{K}$. Hence,

$$
\angle\left(\mathbf{n}_{p}, \mathbf{n}_{q}\right) \leq \int_{0}^{\xi}\left\|S \gamma^{\prime}(s)\right\| d s \leq \mathcal{K} \xi \leq \mathcal{K} \epsilon \leq \frac{1}{2} .
$$

Writing $q=\left(q_{1}, q_{2}, q_{n}\right)$ with respect to the Darboux frame centered at $p=(0,0,0)$, it follows that

$$
\left\|D h\left(q_{1}, q_{2}\right)\right\|=\tan \angle\left(\mathbf{n}_{p}, \mathbf{n}_{q}\right) \leq \tan \frac{1}{2}
$$

Plugging this into (17), we find that $\left|D_{\mathbf{v}}^{2} h\right| \leq 2 \mathcal{K}$, which, together with (14), yields the first part of the lemma.

In order to prove the second part, we first note that $D^{2} h(0)=D^{2} P(0)$ and that $D^{2} P$ is constant. Hence,

$$
\left|h\left(e_{1}, e_{2}\right)-P\left(e_{1}, e_{2}\right)\right|=\left|\int_{0}^{d} \int_{0}^{t} \int_{0}^{\tau} D_{\mathbf{v}}^{3} h(\sigma \mathbf{v}) d \sigma d \tau d t\right| .
$$

We consequently seek a bound for $\left|D_{\mathbf{v}}^{3} h\right|$ in terms of $\mathcal{K}$ and $\mathcal{K}^{\prime}$. Considering (16) and taking another derivative with respect to $\mathbf{v}$ yields

$$
\begin{aligned}
D_{\mathbf{v}}^{3} h= & D_{\mathbf{v}}\left(\kappa_{\mathbf{v}}\right) \sqrt{1+\|D h\|^{2}} \mathrm{I}(\mathbf{v}, \mathbf{v}) \\
& +\kappa_{\mathbf{v}} D_{\mathbf{v}}\left(\sqrt{1+\|D h\|^{2}}\right) \mathrm{I}(\mathbf{v}, \mathbf{v}) \\
& +\kappa_{\mathbf{v}} \sqrt{1+\|D h\|^{2}} D_{\mathbf{v}}(\mathrm{I}(\mathbf{v}, \mathbf{v})) .
\end{aligned}
$$

We bound the terms appearing on the right-hand side one by one. To treat the first term, we use (16) and $\nabla \mathrm{I} \equiv 0$, where $\nabla$ denotes covariant differentiation with respect to the metric induced by $\mathrm{I}$, to derive

$$
D_{\mathbf{v}} \kappa_{\mathbf{v}}=\frac{\mathrm{I}\left(\left(\nabla_{\mathbf{v}} S\right) \mathbf{v}, \mathbf{v}\right)+2 \mathrm{I}\left(S \mathbf{v}, \nabla_{\mathbf{v}} \mathbf{v}\right)-2 \kappa_{\mathbf{v}} \mathrm{I}\left(\mathbf{v}, \nabla_{\mathbf{v}} \mathbf{v}\right)}{\mathrm{I}(\mathbf{v}, \mathbf{v})} .
$$

Let $\mathbf{u}(x, y)=\mathbf{u}$ be a constant vector field in the parameter domain. Using the Koszul formula for the Levi-Civita connection and applying (15) yields

$$
2 \mathrm{I}\left(\nabla_{\mathbf{v}} \mathbf{v}, \mathbf{u}\right)=2 \mathbf{v}(\mathrm{I}(\mathbf{v}, \mathbf{u}))-\mathbf{u}(\mathrm{I}(\mathbf{v}, \mathbf{v}))=2 D_{\mathbf{v}}^{2} h D_{\mathbf{u}} h .
$$

The last equality only depends on the value of $\mathbf{u}(x, y)$ at the point $(x, y)$ and therefore holds for any field $\mathbf{u}$ in the parameter domain. From (17), (18), and $\|\mathbf{v}\|=1$ we obtain

$$
\left|\mathrm{I}\left(\nabla_{\mathbf{v}} \mathbf{v}, \mathbf{u}\right)\right| \leq C\|\mathbf{u}\|
$$


Fig. 8 The discrete curvature vector $k_{\mathbf{e}}$ is the sum of $\mathrm{J}_{\mathbf{f}} \mathbf{e}$ (the rotation of $\mathbf{e}$ by $\frac{\pi}{2}$ about the axis $\mathbf{e} \times \mathbf{f}$ ) and $\mathrm{J}_{\mathbf{g}} \mathbf{e}$ (the rotation of $\mathbf{e}$ by $\frac{\pi}{2}$ about the axis $\mathbf{e} \times \mathbf{g}$ )

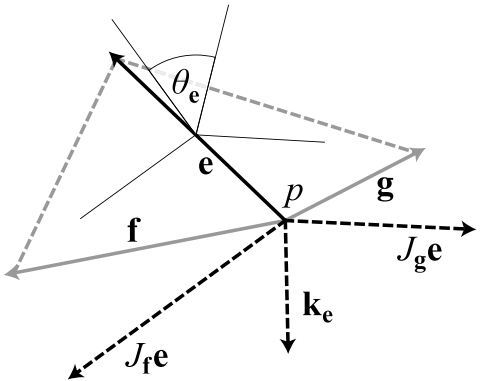

with $C=C(\mathcal{K})$. This can be used in (21), together with our bounds on the norms of $S$ and $\nabla S$ in terms of $\mathcal{K}$ and $\mathcal{K}^{\prime}$, respectively, to obtain an upper bound for the first term in (20) by $C\left(\mathcal{K}, \mathcal{K}^{\prime}\right)$. Bounds for the remaining two terms in (20) can be obtained in a similar fashion using (15) and (16), proving the estimate $\left|D_{\mathbf{v}}^{3} h\right| \leq C\left(\mathcal{K}, \mathcal{K}^{\prime}\right)$. Using (19) and $d \leq \epsilon$ then implies the claim of the second part in the statement of the lemma.

Corollary 14 With the same assumptions as in Lemma 13 and by defining $\delta_{\kappa}=$ $\kappa_{2}-\kappa_{1}$, we obtain

$$
e_{n}=\frac{\kappa_{1}}{2} \mathbf{e}^{2}+\frac{\delta_{\kappa}}{2} e_{2}^{2}+O\left(\epsilon^{3}\right)=\frac{\kappa_{2}}{2} \mathbf{e}^{2}-\frac{\delta_{\kappa}}{2} e_{1}^{2}+O\left(\epsilon^{3}\right)
$$

with $\left|O\left(\epsilon^{3}\right)\right| \leq C\left(\mathcal{K}, \mathcal{K}^{\prime}\right) \epsilon^{3}$.

Proof From the second part of Lemma 13 and simple algebraic manipulations we deduce that

$$
e_{n}=\frac{\kappa_{1}}{2} \mathbf{e}^{2}-\frac{\kappa_{1}}{2} e_{n}^{2}+\frac{\delta_{\kappa}}{2} e_{2}^{2}+O\left(\epsilon^{3}\right)=\frac{\kappa_{1}}{2} \mathbf{e}^{2}+\frac{\delta_{\kappa}}{2} e_{2}^{2}+O\left(\epsilon^{3}\right) .
$$

The last equality follows from Lemma 13 and the sampling condition $\epsilon \leq \frac{1}{2 \mathcal{K}}$ (which is implied by our basic assumptions), which together ensure that $e_{n}^{2} \leq \mathcal{K}^{2} \epsilon^{4} \leq \frac{\mathcal{K}}{2} \epsilon^{3}$. The second equation in the statement of the corollary follows analogously.

For the following discussion, it will be useful to work with the curvature vector $\mathbf{k}_{\mathbf{e}}$ corresponding to the discrete integrated curvature $k_{\mathbf{e}}=2 \sin \frac{\theta_{\mathbf{e}}}{2}\|\mathbf{e}\|$ (see Sect. 2.1). With our usual notions, consider the two edge vectors $\mathbf{f}$ and $\mathbf{g}$ directly neighboring $\mathbf{e}$ in $s t(p)$ (see Fig. 8). Recall from Corollary 9 that we may assume that $(\mathbf{e} \times \mathbf{f}) \cdot \mathbf{n}>0$ and $(\mathbf{e} \times \mathbf{g}) \cdot \mathbf{n}<0$, where $\mathbf{n}$ is the normal of $M$ at $p$. A straightforward calculation reveals that we can express the discrete curvature vector by

$$
\mathbf{k}_{\mathbf{e}}=\mathrm{J}_{\mathbf{f}} \mathbf{e}+\mathrm{J}_{\mathbf{g}} \mathbf{e},
$$

where $\mathrm{J}_{\mathbf{f}}$ and $\mathrm{J}_{\mathbf{g}}$ denote the rotations by $\frac{\pi}{2}$ around the axes $\mathbf{e} \times \mathbf{f}$ and $\mathbf{e} \times \mathbf{g}$, respectively (see Fig. 8). Consider now the splitting

$$
\mathbf{k}_{\mathbf{e}}=\left(\mathbf{k}_{\mathbf{e}}\right)_{t}+\left(\mathbf{k}_{\mathbf{e}}\right)_{n}
$$


of $\mathbf{k}_{\mathbf{e}}$ into its tangential and normal component with respect to $T_{p} M$. We show that the tangential component is negligible:

Lemma 15 Under the same assumptions as in Lemma 13, the projection of the discrete curvature vector $\mathbf{k}_{\mathbf{e}}$ onto the tangent plane $T_{p} M$ satisfies $\left\|\left(\mathbf{k}_{\mathbf{e}}\right)_{t}\right\| \leq 10 \mathcal{K}^{2} \rho^{2} \epsilon^{3}$.

Proof With the notions and assumption of the preceding discussion, we first note that a straightforward calculation reveals that

$$
\mathrm{J}_{\mathbf{f}} \mathbf{e}=\frac{\mathbf{e}^{2} \mathbf{f}-(\mathbf{f} \cdot \mathbf{e}) \mathbf{e}}{\|\mathbf{e} \times \mathbf{f}\|}
$$

and analogously for $\mathrm{J}_{\mathrm{g}} \mathbf{e}$.

With respect to $T_{p} M$, let $\mathbf{e}_{t}, \mathbf{f}_{t}$, and $\mathbf{g}_{t}$ denote the tangential components of the vectors $\mathbf{e}, \mathbf{f}$, and $\mathbf{g}$, respectively. Then, by assumption, we have $\left(\mathbf{e}_{t} \times \mathbf{f}_{t}\right) \cdot \mathbf{n}>0$ and $\left(\mathbf{e}_{t} \times \mathbf{g}_{t}\right) \cdot \mathbf{n}<0$. The tangential part of the discrete curvature vector $\mathbf{k}_{\mathbf{e}}$ is given by $\left(\mathbf{k}_{\mathbf{e}}\right)_{t}=\left(\mathbf{J}_{\mathbf{g}} \mathbf{e}\right)_{t}+\left(\mathbf{J}_{\mathbf{f}} \mathbf{f}\right)_{t}$, where we deduce from (23), using the coordinates of our fixed Darboux frame at $p$, that

$$
\left(\mathrm{J}_{\mathbf{f}} \mathbf{e}\right)_{t}=\frac{\|\mathbf{e}\|^{2} \mathbf{f}_{t}-(\mathbf{f} \cdot \mathbf{e}) \mathbf{e}_{t}}{\|\mathbf{e} \times \mathbf{f}\|}=\frac{\left\|\mathbf{e}_{t} \times \mathbf{f}_{t}\right\|}{\|\mathbf{e} \times \mathbf{f}\|}\left(-e_{2}, e_{1}, 0\right)+\frac{e_{n}^{2} \mathbf{f}_{t}-e_{n} f_{n} \mathbf{e}_{t}}{\|\mathbf{e} \times \mathbf{f}\|}
$$

and similarly

$$
\left(\mathrm{J}_{\mathbf{g}} \mathbf{e}\right)_{t}=-\frac{\left\|\mathbf{e}_{t} \times \mathbf{g}_{t}\right\|}{\|\mathbf{e} \times \mathbf{g}\|}\left(-e_{2}, e_{1}, 0\right)+\frac{e_{n}^{2} \mathbf{g}_{t}-e_{n} g_{n} \mathbf{e}_{t}}{\|\mathbf{e} \times \mathbf{g}\|} .
$$

Using the first part of Lemma 13 and the definition of shape regularity (9), we obtain

$$
\begin{aligned}
\|\mathbf{e} \times \mathbf{f}\|^{2} & =\left\|\mathbf{e}_{t} \times \mathbf{f}_{t}\right\|^{2}+\left(e_{n} f_{1}-e_{1} f_{n}\right)^{2}+\left(e_{n} f_{2}-e_{2} f_{n}\right)^{2} \\
& \leq\left\|\mathbf{e}_{t} \times \mathbf{f}_{t}\right\|^{2}+8(\mathcal{K}\|\mathbf{e}\|\|\mathbf{f}\| \epsilon)^{2} \\
& \leq\left\|\mathbf{e}_{t} \times \mathbf{f}_{t}\right\|^{2}+8 \mathcal{K}^{2} \rho^{2} \epsilon^{2}\|\mathbf{e} \times \mathbf{f}\|^{2}
\end{aligned}
$$

Therefore, we have

$$
1 \geq \frac{\left\|\mathbf{e}_{t} \times \mathbf{f}_{t}\right\|}{\|\mathbf{e} \times \mathbf{f}\|} \geq \frac{\left\|\mathbf{e}_{t} \times \mathbf{f}_{t}\right\|^{2}}{\|\mathbf{e} \times \mathbf{f}\|^{2}} \geq 1-8 \mathcal{K}^{2} \rho^{2} \epsilon^{2},
$$

and similarly for $\mathbf{g}$. It follows that the two terms in $\left(\mathrm{J}_{\mathbf{f}} \mathbf{e}+\mathrm{J}_{\mathbf{g}} \mathbf{e}\right)_{t}$ containing $\left(-e_{2}, e_{1}, 0\right)$ cancel up to a term bounded by $\left(8 \mathcal{K}^{2} \rho^{2}\right) \epsilon^{3}$, where the power of three is due to the fact that the norm of $\left(-e_{2}, e_{1}, 0\right)$ is also bounded by $\epsilon$. Moreover, we observe that the first part of Lemma 13 and the definition of shape regularity (9) yield

$$
\frac{\left\|e_{n}^{2} \mathbf{f}_{t}-e_{n} f_{n} \mathbf{e}_{t}\right\|}{\|\mathbf{e} \times \mathbf{f}\|} \leq \frac{\mathcal{K}^{2}\|\mathbf{e}\|^{4}\|\mathbf{f}\|+\mathcal{K}^{2}\|\mathbf{e}\|^{3}\|\mathbf{f}\|^{2}}{\|\mathbf{e} \times \mathbf{f}\|} \leq 2 \mathcal{K}^{2} \rho \epsilon^{3},
$$


and analogously for $\mathbf{g}$. Therefore, we arrive at

$$
\left\|\left(\mathbf{k}_{\mathbf{e}}\right)_{t}\right\|=\left\|\left(\mathbf{J}_{\mathbf{f}} \mathbf{e}+\mathbf{J}_{\mathbf{g}} \mathbf{e}\right)_{t}\right\| \leq\left(10 \mathcal{K}^{2} \rho^{2}\right) \epsilon^{3},
$$

proving the claim.

The above implies the main result of this section:

Proof of Proposition 12 To estimate the normal component $\left(\mathbf{k}_{\mathbf{e}}\right)_{n}=\left(\mathrm{J}_{\mathbf{f}} \mathbf{e}\right)_{n}+\left(\mathrm{J}_{\mathbf{g}} \mathbf{e}\right)_{n}$ of the discrete curvature vector, we use (23) to obtain

$$
\left(\mathrm{J}_{\mathbf{f}} \mathbf{e}\right)_{n}=\frac{\mathbf{e}^{2} f_{n}-(\mathbf{f} \cdot \mathbf{e}) e_{n}}{\|\mathbf{e} \times \mathbf{f}\|},
$$

and analogously for $\left(\mathrm{J}_{\mathbf{g}} \mathbf{e}\right)_{n}$.

We note that the circumcentric area of $\mathbf{e}$ can be expressed in a similar manner as a $\operatorname{sum} A_{\mathbf{e}}=A_{\mathbf{e}, \mathbf{f}}+A_{\mathbf{e}, \mathbf{g}}$, where

$$
A_{\mathbf{e}, \mathbf{f}}=\frac{\mathbf{f} \cdot(\mathbf{f}-\mathbf{e})}{4\|\mathbf{e} \times \mathbf{f}\|} \mathbf{e}^{2},
$$

and analogously for $A_{\mathbf{e}, \mathbf{g}}$. Applying Corollary 14 , we obtain

$$
\begin{aligned}
\left(\mathrm{J}_{\mathbf{f}} \mathbf{e}\right)_{n} & =\frac{\mathbf{e}^{2}\left(\kappa_{2} \mathbf{f}^{2}-\delta_{\kappa} f_{1}^{2}\right)-\mathbf{f} \cdot \mathbf{e}\left(\kappa_{2} \mathbf{e}^{2}-\delta_{\kappa} e_{1}^{2}\right)}{2\|\mathbf{e} \times \mathbf{f}\|}+O\left(\epsilon^{3}\right) \\
& =2 A_{\mathbf{e}, \mathbf{f}} \kappa_{2}-\delta_{\kappa} f_{1}^{2} \frac{\mathbf{e}^{2}}{2\|\mathbf{e} \times \mathbf{f}\|}-\delta_{\kappa} \mathbf{f} \cdot \mathbf{e} \frac{e_{1}^{2}}{2\|\mathbf{e} \times \mathbf{f}\|}+O\left(\epsilon^{3}\right),
\end{aligned}
$$

and analogously for $\left(\mathrm{J}_{\mathbf{g}} \mathbf{e}\right)_{n}$, with $\left|O\left(\epsilon^{3}\right)\right| \leq C\left(\mathcal{K}, \mathcal{K}^{\prime}, \rho\right) \epsilon^{3}$. Applying the shape regularity condition (9) yields

$$
\left|\left(\mathrm{J}_{\mathbf{f}} \mathbf{e}\right)_{n}-2 A_{\mathbf{e}, \mathbf{f}} \kappa_{2}\right| \leq C\left(\left|\delta_{\kappa}\left(f_{1}^{2}+\mathbf{f} \cdot \mathbf{e}\right)\right|+\epsilon^{3}\right),
$$

and similarly for the difference between $\left(\mathrm{J}_{\mathbf{g}} \mathbf{e}\right)_{n}$ and $2 A_{\mathbf{e}, \mathbf{g}} \kappa_{2}$. According to the statement of Proposition 12, we assume that $\mathbf{e}$ is associated with the direction $\mathbf{v}_{1}$, whereas $\mathbf{f}$ and $\mathbf{g}$ are associated with $\mathbf{v}_{2}$. Using our notion of tangential deviation from Definition 4 and noting that $|\mathbf{f} \cdot \mathbf{e}| \leq\left|e_{2}+f_{1}\right| \epsilon+O\left(\epsilon^{4}\right)$, we arrive at

$$
\left|\left(\mathbf{k}_{\mathbf{e}}\right)_{n}-2 A_{\mathbf{e}} \kappa_{2}\right| \leq C\left(\left|\delta_{\kappa}\left(e_{d}+f_{d}+g_{d}\right)\right| \epsilon+\epsilon^{3}\right),
$$

which implies the claim, since $\left(\mathbf{k}_{\mathbf{e}}\right)_{t}$ is of order $O\left(\epsilon^{3}\right)$ by Lemma 15 .

\subsection{Estimates for Nonumbilical Vertices}

As mentioned before, the results of the preceding section are not entirely specific for nets of curvature lines but hold for a larger class of smoothly embedded nets. As such, these results do not suffice to prove our main error estimate in Theorem 2, due to the failure of uniform convergence of curvature approximations constructed 
in a 1-local manner for general nets. Indeed, assuming that $\mathbf{e}$ is associated with the principal direction $\mathbf{v}_{1}$, we seek a bound of the form

$$
\left|k_{\mathbf{e}}-2 A_{\mathbf{e}} \kappa_{2}\right| \leq C \epsilon^{3},
$$

from which we may derive the desired main error estimate by employing our results on the existence of uniform lower and upper bounds of (sufficiently many) circumcentric areas (see Sect. 3.1).

While a bound of the form (24) does not hold for general smooth nets, it is indeed valid for nets of curvature lines. This is a consequence of the results of the preceding section and the fact that for nets of curvature lines, we have

$$
\left|\delta_{\kappa}(p) e_{d}\right| \leq C \epsilon^{2}
$$

which is trivially satisfied for umbilical vertices and true for nonumbilical ones, provided that we associate e with its canonical principal direction. This is precisely the main result of this section.

Observe that there is a simple case where (25) is obviously fulfilled: let $M$ be a paraboloid, and let $p$ be its apex. Consider the four edge vectors emanating from $p$ in a local polyhedral approximation of a net of curvature lines containing $p$ as a vertex. Then the tangential deviation of each of these edges vanishes, so (25) is clearly satisfied.

The main difference between nets of curvature lines on arbitrary smooth surfaces and the specific case of a paraboloid is the fact that curvature lines usually have nonzero geodesic curvature $\kappa^{g}$. While the tangential deviation $e_{d}$ can always be bounded by $C \epsilon^{2}$, with $C=C\left(\mathcal{K}, \kappa^{g}\right)$, this does not suffice for a uniform error bound, since $\kappa^{g}$ may blow up at umbilical points. Perhaps surprisingly, though, the product $\left|\delta_{\kappa}(p) e_{d}\right|$ can be bounded for nets of curvature lines:

Proposition 16 Let $\mathbf{e}$ be an edge vector emanating from a nonumbilical vertex $p$ in a local polyhedral approximation of a discrete net of curvature lines on M. If $\mathbf{e}$ is associated with its canonical principal direction, then our basic assumptions imply

$$
\left|\delta_{\kappa}(p) e_{d}\right| \leq\left(\mathcal{K}^{2}+4 \mathcal{K}^{\prime}\right) \epsilon^{2},
$$

where $e_{d}$ is the tangential deviation of $\mathbf{e}$, and $\mathcal{K}, \mathcal{K}^{\prime}$ denote our usual bounds on normal curvatures and their derivatives, respectively.

The intuition behind this statement is as follows. Roughly, $e_{d}$ is proportional to the geodesic curvature of the curvature line corresponding to e. This geodesic curvature, in turn, is inversely proportional to $\delta_{\kappa}$, as the next lemma shows. Therefore, the product $\delta_{\kappa} e_{d}$ can be uniformly bounded.

Lemma 17 At any nonumbilical point of $M$, the geodesic curvature $\kappa_{1}^{g}$ of the principal curvature line along $\mathbf{v}_{1}$ satisfies

$$
\kappa_{1}^{g}=\frac{\nabla_{\mathbf{v}_{2}} \kappa_{1}}{\kappa_{1}-\kappa_{2}} \quad \text { and thus } \quad\left|\kappa_{1}^{g}\right| \leq \frac{\mathcal{K}^{\prime}}{\left|\kappa_{1}-\kappa_{2}\right|},
$$


where $\kappa_{1}$ and $\kappa_{2}$ denote the principal curvatures corresponding to the principal directions $\mathbf{v}_{1}$ and $\mathbf{v}_{2}$, respectively.

Proof Since $\mathbf{v}_{1}$ and $\mathbf{v}_{2}$ are orthonormal eigenvectors of the shape operator $S$, we have $S \mathbf{v}_{1} \cdot \mathbf{v}_{2}=0$. We use the Frenet formulas

$$
\begin{aligned}
& \nabla_{\mathbf{v}_{1}} \mathbf{v}_{1}=\kappa_{1}^{g} \mathbf{v}_{2}, \quad \nabla_{\mathbf{v}_{1}} \mathbf{v}_{2}=-\kappa_{1}^{g} \mathbf{v}_{1}, \\
& \nabla_{\mathbf{v}_{2}} \mathbf{v}_{1}=\kappa_{2}^{g} \mathbf{v}_{2}, \quad \text { and } \quad \nabla_{\mathbf{v}_{2}} \mathbf{v}_{2}=-\kappa_{2}^{g} \mathbf{v}_{1}
\end{aligned}
$$

and the Codazzi-Mainardi equation

$$
\left(\nabla_{\mathbf{u}} S\right) \mathbf{v}=\left(\nabla_{\mathbf{v}} S\right) \mathbf{u}
$$

to obtain

$$
\begin{aligned}
0 & =\nabla_{\mathbf{v}_{1}}\left(S \mathbf{v}_{1} \cdot \mathbf{v}_{2}\right)=\nabla_{\mathbf{v}_{1}}\left(S \mathbf{v}_{2} \cdot \mathbf{v}_{1}\right) \\
& =\left(\nabla_{\mathbf{v}_{1}} S\right) \mathbf{v}_{2} \cdot \mathbf{v}_{1}+S\left(\nabla_{\mathbf{v}_{1}} \mathbf{v}_{2}\right) \cdot \mathbf{v}_{1}+S \mathbf{v}_{2} \cdot \nabla_{\mathbf{v}_{1}} \mathbf{v}_{1} \\
& =\left(\nabla_{\mathbf{v}_{2}} S\right) \mathbf{v}_{1} \cdot \mathbf{v}_{1}+S\left(-\kappa_{1}^{g} \mathbf{v}_{1}\right) \cdot \mathbf{v}_{1}+S \mathbf{v}_{2} \cdot\left(\kappa_{1}^{g} \mathbf{v}_{2}\right) \\
& =\left(\nabla_{\mathbf{v}_{2}} S\right) \mathbf{v}_{1} \cdot \mathbf{v}_{1}+\kappa_{1}^{g}\left(-\kappa_{1} \mathbf{v}_{1} \cdot \mathbf{v}_{1}+\kappa_{2} \mathbf{v}_{2} \cdot \mathbf{v}_{2}\right) \\
& =\nabla_{\mathbf{v}_{2}}\left(S \mathbf{v}_{1} \cdot \mathbf{v}_{1}\right)-S\left(\nabla_{\mathbf{v}_{2}} \mathbf{v}_{1}\right) \cdot \mathbf{v}_{1}-S \mathbf{v}_{1} \cdot \nabla_{\mathbf{v}_{2}} \mathbf{v}_{1}+\kappa_{1}^{g}\left(\kappa_{2}-\kappa_{1}\right) \\
& =\nabla_{\mathbf{v}_{2}}\left(S \mathbf{v}_{1} \cdot \mathbf{v}_{1}\right)-S\left(\kappa_{2}^{g} \mathbf{v}_{2}\right) \cdot \mathbf{v}_{1}-S \mathbf{v}_{1} \cdot \kappa_{2}^{g} \mathbf{v}_{2}+\kappa_{1}^{g}\left(\kappa_{2}-\kappa_{1}\right) \\
& =\nabla_{\mathbf{v}_{2}} \kappa_{1}+\kappa_{1}^{g}\left(\kappa_{2}-\kappa_{1}\right),
\end{aligned}
$$

proving the first part. The second part follows from the definition of $\mathcal{K}^{\prime}$.

Proof of Proposition 16 Let $\gamma:[0, \epsilon] \rightarrow M$ be the curvature line that is canonically associated with e, parameterized by arc-length and passing through $p=\gamma(0)$. By definition, $e_{d}$ is bounded above by the maximum distance from $\gamma$ to the tangent line passing trough $\gamma^{\prime}(0)$. Since $\gamma$ is parameterized by arc-length, we obtain

$$
e_{d} \leq \frac{\mathcal{K}_{\gamma}}{2} \epsilon^{2}
$$

where $\mathcal{K}_{\gamma}$ denotes the maximum curvature of $\gamma$ as a space curve. Decomposing the curvature vector of $\gamma$ into its normal and geodesic components, and denoting by $\mathcal{K}_{\gamma}^{n}$ and $\mathcal{K}_{\gamma}^{g}$ the respective maxima of the norms of these components, Lemma 17 yields

$$
\mathcal{K}_{\gamma} \leq \mathcal{K}_{\gamma}^{n}+\mathcal{K}_{\gamma}^{g} \leq \mathcal{K}+\max _{s \in[0, \epsilon]} \frac{\mathcal{K}^{\prime}}{\left|\delta_{\mathcal{K}}(\gamma(s))\right|},
$$

since $\mathcal{K}_{\gamma}^{n} \leq \mathcal{K}$ by the definition of $\mathcal{K}$. Consequently, we seek a lower bound for $\left|\delta_{K}(\gamma(s))\right|$. To do so, first observe that by the definition of $\mathcal{K}^{\prime}$ the derivatives of $\kappa_{1}$ and $\kappa_{2}$ are bounded by $\mathcal{K}^{\prime}$. Hence, the function $\delta_{\kappa}$ is Lipschitz with constant $2 \mathcal{K}^{\prime}$, i.e.,

$$
\left|\delta_{\kappa}(p)-\delta_{\kappa}(q)\right| \leq 2 \mathcal{K}^{\prime} d_{M}(p, q)
$$

for every point $q \in M$. 
We now distinguish two cases: (i) $\left|\delta_{\kappa}(p)\right|<4 \mathcal{K}^{\prime} \epsilon$ and (ii) $\left|\delta_{\kappa}(p)\right| \geq 4 \mathcal{K}^{\prime} \epsilon$. In the first case, we immediately obtain

$$
\left|\delta_{\kappa}(p) e_{d}\right| \leq 4 \mathcal{K}^{\prime} \epsilon^{2}
$$

which already proves the claim of the lemma. In the second case, we observe that for all $s \in[0, \epsilon]$, we have

$$
\left|\delta_{\kappa}(\gamma(s))\right| \geq\left|\delta_{\kappa}(p)\right|-2 \mathcal{K}^{\prime} \epsilon \geq \frac{1}{2}\left|\delta_{\kappa}(p)\right| .
$$

Plugging this into (27) gives

$$
\mathcal{K}_{\gamma} \leq \mathcal{K}+\frac{2 \mathcal{K}^{\prime}}{\left|\delta_{\kappa}(p)\right|}
$$

Together with (26), this yields

$$
\left|\delta_{\kappa}(p) e_{d}\right| \leq\left|\delta_{\kappa}(p)\right|\left(\frac{\mathcal{K}}{2}+\frac{\mathcal{K}^{\prime}}{\left|\delta_{\kappa}(p)\right|}\right) \epsilon^{2} \leq\left(\mathcal{K}^{2}+\mathcal{K}^{\prime}\right) \epsilon^{2},
$$

completing the proof.

\subsection{Combining the Strings}

We are now in the position to prove Theorem 2.

To this end, assume that the edge $\mathbf{e}$ in our local polyhedral approximation is associated with the principal direction given by $\mathbf{v}_{1}$, and let this be the canonical direction if $p$ is not umbilical. Furthermore, let $k_{\mathbf{e}}=2 \sin \frac{\theta_{\mathbf{e}}}{2}\|\mathbf{e}\|$ be the discrete integrated curvature introduced in Sect. 2.1. Then Propositions 12 and 16 imply that there exists a constant $C=C\left(\mathcal{K}, \mathcal{K}^{\prime}, \rho\right)$ such that

$$
\left|k_{\mathbf{e}}-2 A_{\mathbf{e}} \kappa_{2}\right| \leq C \epsilon^{3} .
$$

Assume additionally that $\mathbf{e}$ is chosen such that it maximizes the circumcentric area $A_{\mathbf{e}}$. (There are exactly two choices for nonumbilical vertices.) Proposition 3 shows that this choice leads to lower and upper bounds for $A_{\mathbf{e}}$ by $(1 / C) \epsilon^{2}$ and $C \epsilon^{2}$, respectively, with $C=C(\rho)$. Therefore, we can divide (28) by $2 A_{\mathbf{e}}$ to obtain

$$
\left|\frac{k_{\mathbf{e}}}{2 A_{\mathbf{e}}}-\kappa_{2}\right| \leq C \epsilon
$$

with $C=C\left(\mathcal{K}, \mathcal{K}^{\prime}, \rho\right)$.

Finally, in order to prove our error estimate for the two other edge-based integrated discrete curvatures, $k_{\mathbf{e}}=2 \tan \frac{\theta_{\mathbf{e}}}{2}\|\mathbf{e}\|$ and $k_{\mathbf{e}}=\theta_{\mathbf{e}}\|\mathbf{e}\|$, we infer from Lemma 8 and a simple application of Taylor's theorem that there exists a constant $C=C(\mathcal{K}, \rho)$ such that

$$
\left|\theta_{\mathbf{e}}-2 \sin \frac{\theta_{\mathbf{e}}}{2}\right| \leq C \epsilon^{3} \text { and } \quad\left|\theta_{\mathbf{e}}-2 \tan \frac{\theta_{\mathbf{e}}}{2}\right| \leq C \epsilon^{3},
$$


which can be used to obtain the requisite bound (28) for the other two discrete curvature definitions as well. This completes the proof of Theorem 2.

Acknowledgements We would like to thank the anonymous reviewers for their very valuable and detailed comments. We also thank Emanuel Huhnen-Venedey and Ramsay Dyer for their helpful feedback. This work was partially supported by the DFG Research Center MATHEON and the DFG Research Unit Polyhedral Surfaces.

Open Access This article is distributed under the terms of the Creative Commons Attribution Noncommercial License which permits any noncommercial use, distribution, and reproduction in any medium, provided the original author(s) and source are credited.

\section{References}

1. Belkin, M., Sun, J., Wang, Y.: Discrete Laplace operator on meshed surfaces. In: Proceedings of the twenty-fourth annual symposium on Computational geometry, pp. 278-287 (2008)

2. Berry, M.V., Hannay, J.H.: Umbilic points on Gaussian random surfaces. J. Phys. A: Math. Gen. 10, 1809-1821 (1977)

3. Bobenko, A., Springborn, B.: A discrete Laplace-Beltrami operator for simplicial surfaces. Discrete Comput. Geom. 38(4), 740-756 (2007)

4. Bobenko, A.I., Suris, Y.: Discrete time Lagrangian mechanics on Lie groups, with an application to the Lagrange top. Commun. Math. Phys. 204(1), 147-188 (1999)

5. Bobenko, A.I., Suris, Y.B.: Discrete Differential Geometry: Integrable Structure. Graduate Studies in Mathematics, vol. 98. American Mathematical Society, Providence (2008)

6. Bobenko, A., Schröder, P., Sullivan, J., Ziegler, G. (eds.): Discrete Differential Geometry. Oberwolfach Seminars, vol. 38. Birkhäuser, Basel (2008)

7. Cazals, F., Pouget, M.: Estimating differential quantities using polynomial fitting of osculating jets. Comput. Aided Geom. Des. 22(2), 121-146 (2005)

8. Cohen-Steiner, D., Morvan, J.-M.: Second fundamental measure of geometric sets and local approximation of curvatures. J. Differ. Geom. 73(3), 363-394 (2006)

9. Desbrun, M., Hirani, A.N., Leok, M., Marsden, J.E.: Discrete exterior calculus. Arxiv preprint (2005). arXiv:math/0508341

10. Dyer, R., Schaefer, S.: Circumcentric dual cells with negative area. Technical Report TR 2009-02, School of Computing Science, Simon Fraser University, Burnaby, BC, Canada (2009)

11. Dziuk, G.: Finite elements for the Beltrami operator on arbitrary surfaces. In: Partial Differential Equations and Calculus of Variations. Lecture Notes in Mathematics, vol. 1357, pp. 142-155. Springer, Berlin (1988)

12. Federer, H.: Curvature measures. Trans. Am. Math. 93(3), 418-491 (1959)

13. Fu, J.H.G.: Convergence of curvatures in secant approximations. J. Differ. Geom. 37, 177-190 (1993)

14. Grinspun, E., Desbrun, M. (eds.): Discrete Differential Geometry: An Applied Introduction. ACM SIGGRAPH Courses Notes. ACM, New York (2006)

15. Hildebrandt, K., Polthier, K., Wardetzky, M.: On the convergence of metric and geometric properties of polyhedral surfaces. Geom. Dedic. 123(1), 89-112 (2006)

16. Hoffmann, T.: Discrete Hashimoto surfaces and a doubly discrete smoke-ring flow. In: Discrete Differential Geometry. Oberwolfach Seminars, vol. 38, pp. 95-116. Birkhäuser, Basel (2008)

17. Meek, D.S., Walton, D.J.: On surface normal and Gaussian curvature approximations given data sampled from a smooth surface. Comput. Aided Geom. Des. 17(6), 521-543 (2000)

18. Morvan, J.-M., Thibert, B.: Approximation of the normal vector field and the area of a smooth surface. Discrete Comput. Geom. 32(3), 383-400 (2004)

19. Pinkall, U., Polthier, K.: Computing discrete minimal surfaces and their conjugates. Exp. Math. 2(1), 15-36 (1993)

20. Steiner, J.: Ueber parallele Flächen. Monatsbericht der Akademie der Wissenschaften zu Berlin, pp. 114-118 (1840)

21. Sullivan, J.M.: Curves of finite total curvature. In: Discrete Differential Geometry. Oberwolfach Seminars, vol. 38, pp. 137-162. Birkhäuser, Basel (2008) 
22. Wintgen, P.: Normal cycle and integral curvature for polyhedra in Riemannian manifolds. In: Soós, Szenthe (eds.) Differential Geometry, pp. 805-816. North-Holland, Amsterdam (1982)

23. $\mathrm{Xu}, \mathrm{G} .:$ Discrete Laplace-Beltrami operator on sphere and optimal spherical triangulations. Int. J. Comput. Geom. Appl. 16(1), 75-93 (2006)

24. Xu, Z., Xu, G., Sun, J.-G.: Convergence analysis of discrete differential geometry operators over surfaces. In: Mathematics of Surfaces XI. LNCS, vol. 3604, pp. 448-457. Springer, Berlin (2005)

25. Zähle, M.: Integral and current representations of Federer's curvature measures. Arch. Math. 46, 557567 (1986) 